\title{
Natural history of severe thrombocytopenia in infectious mononucleosis
}

\author{
Su Yong Wong \\ M.B., Ch.B.
}

\author{
BRUCE BENNETT \\ M.D., F.R.C.P., M.R.C.Path.
}

Department of Medicine, University of Aberdeen, Aberdeen Royal Infirmary

\begin{abstract}
Summary
The natural history of severe thrombocytopenia in two patients with infectious mononucleosis (minimum platelet counts under $10 \times 10^{9}$ and $17 \times 10^{9} / 1$ respectively) is described. In both, the platelet count rose rapidly and spontaneously, reaching approximately $100 \times 10^{9} / 1$ on the seventh day. Bleeding symptoms were also transient and never life-threatening. The possibility of very rapid spontaneous recovery from severe thrombocytopenia must be borne in mind in assessing the effect of any drug in the management of this complication of infectious mononucleosis.
\end{abstract}

\section{Introduction}

While mild thrombocytopenia is common in infectious mononucleosis (IM), severe thrombocytopenia is rare and troublesome haemorrhage is exceedingly rare, occurring in less than $0.05 \%$ of cases (Sharp, 1969; Goldstein and Porter, 1969). Mild thrombocytopenia without bleeding may persist for some weeks but does not require active measures. The natural history of severe thrombocytopenia, when it has occurred, has rarely been described because most recent records are complicated by the administration of steroids which are advocated in the management of thrombocytopenia in IM (Sharp, 1969; Goldstein and Porter, 1969; Weatherall and Bradley, 1974). Lack of data on the natural history of severe thrombocytopenia in IM clearly makes judgement of the efficacy of any drug in its management difficult and the value of these agents in preventing serious haemorrhage in IM is consequently uncertain (Radel and Schorr, 1963). The authors describe two patients with IM presenting with haemorrhagic symptoms and severe thrombocytopenia in both of whom platelet counts rose, without any specific therapy, to $100 \times 10^{\%} / 1$ within 7 days and whose bleeding resolved within even shorter periods.

\section{Case 1}

A 16-year-old girl was admitted with a 24-hr history of nose bleeding and skin bruising. She felt well, was afebrile and had no pharyngitis. She had continuing mild epistaxis, numerous petechiae on legs and trunk with bruising on the forearms; tiny lymph glands were palpable in the neck, and there was no liver or spleen enlargement. The platelet count was under $10 \times 10^{9} / 1, \mathrm{Hb} 12.9 \mathrm{~g} / \mathrm{dl}$, WBC $5 \cdot 7 \times$ $10^{9} / 1$; blood film examination showed $70 \%$ of the white cells to be classical for IM. The IM slide and Paul Bunnell tests were positive and IgM and IgG antibodies to Epstein-Barr (EB) virus were subsequently detected. Steroids were withheld and epistaxis ceased spontaneously within $24 \mathrm{hr}$. Menstruation started on the 2 nd day as expected and continued normally, ceasing on the 6th day. On the 4th day gross pharyngitis with extensive membrane formation, pain and fever developed. Bacterial cultures were always negative, local measures only were employed and the membrane fell off on day 8 when the fever resolved. Epistaxis did not recur and the platelet count rose rapidly and spontaneously to over $100 \times 10^{9} / 1$ on the 7 th day reaching $300 \times$ $10^{9} / 1$ on day 14 (Fig. 1).

\section{Case 2}

A 17-year-old boy had cautery and packing of his right nostril after profuse epistaxis lasting $24 \mathrm{hr}$. On the following day, epistaxis occurred from the left side which was treated similarly and shortly thereafter fever, pharyngitis, cutaneous and mucosal petechiae, lymphadenopathy and splenomegaly were noted. The platelet count was $34 \times 10^{9} / 1$, WBC $7 \cdot 3 \times$ $10^{9} / 1,80 \%$ of the white cell series being typical for IM. The IM slide and Paul Bunnell tests were positive and both IgM and IgG antibodies to EB virus were later demonstrated in the serum. He had received a single dose of $20 \mathrm{mg}$ prednisolone orally on day 2. Steroids were then withheld. Epistaxis continued for a further 2 days and the nose was repacked once. The $\mathrm{Hb}$ fell to $9.4 \mathrm{~g}$ and the platelet count to $17 \times 10^{9} / 1$ on day 3 when he received 3 units fresh blood; bleeding stopped shortly thereafter and the platelet count rose rapidly as shown in 


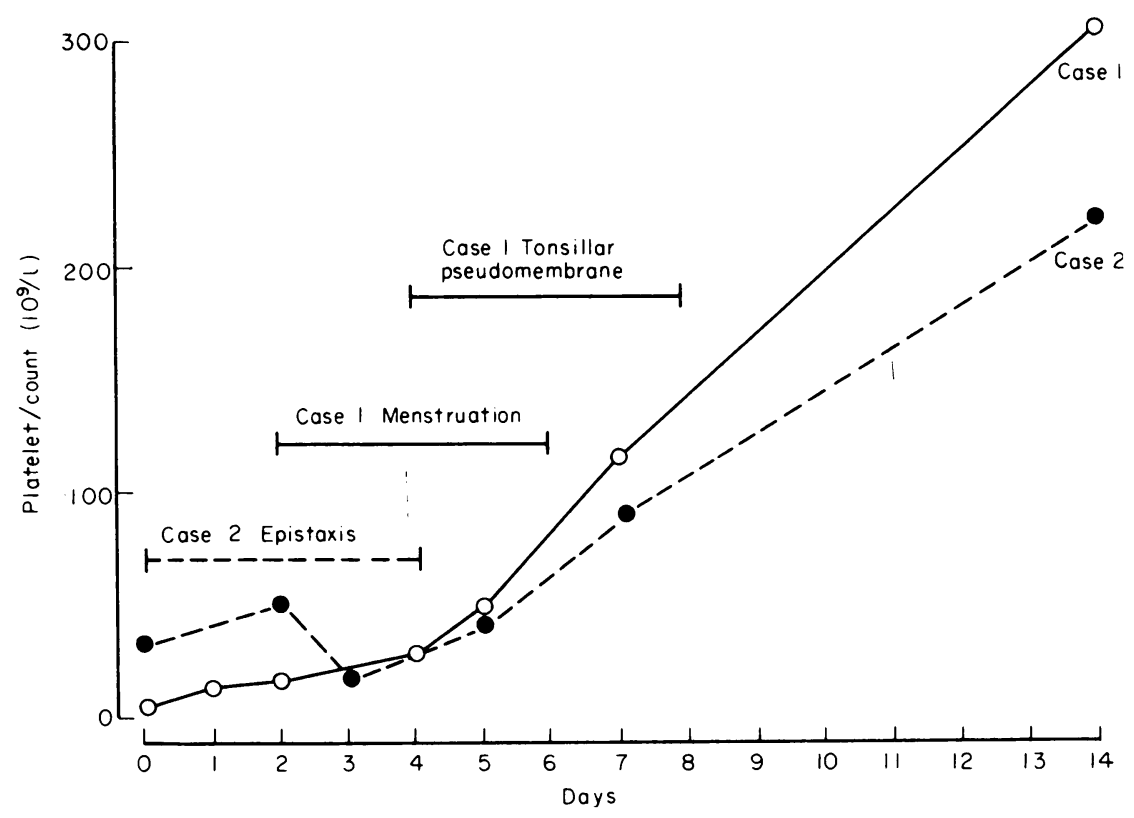

Fig. 1. Platelet counts in two patients with infectious mononucleosis presenting with epistaxis.

Fig. 1, nasal packs being removed on day 5 without further bleeding.

\section{Discussion}

There are few reports of the natural history of severe thrombocytopenic episodes in IM. Some early observations indicated that they might last on occasions for one to several weeks (Angle and Alt, 1950; Benaim, 1952; Finlayson, 1951; Volpe, Sparks and Mautner, 1953; Radel and Schorr, 1963; Clarke and Davies, 1964). Two of the early reports, however, record that rapid spontaneous resolution of severe thrombocytopenia is possible within 4 and 5 days respectively (Goldbloom and Lieberson, 1948; Ogilvie and Parrie, 1952). The two patients described here re-emphasize the point that very rapid spontaneous resolution of severe thrombocytopenia and bleeding symptoms is possible in IM. Although case 2 received a single oral dose of prednisolone on day 2 , his platelet count fell thereafter, and the subsequent rise occurred without steroids.

Steroids have very properly been used in thrombocytopenic IM patients in an attempt to prevent the rare but documented occurrence of dangerous haemorrhage in such patients. Evidence for their efficacy in raising the platelet count varies. Freeman and Wakefield (1958) noted a dramatic rise in platelet count within hours of starting steroid therapy in a severely jaundiced patient with a platelet agglutinin demonstrable in the serum.
Others record rises of the platelet count afte $\stackrel{0}{\overrightarrow{0}}$ steroids marginally faster than those of the prese authors' untreated patients (Myatt and Linn, 195형 Grossman and Wolff, 1959) and there are reports of rises after steroids similar to or slower than those observed in the present patients (Krishnamurthy, Lee and Dosik, 1976; Clarke and Davies, 1964; $\stackrel{\varnothing}{\complement}$ Radel and Schorr, 1963). Perhaps, had the present $\vec{\overrightarrow{ }}$ patients received continuous steroids, the rise in $\frac{5}{3}$ platelet count would have been faster; undoubtedly, had they received steroids and exhibited the spontaneous response recorded here, the authors would have attributed the response to the effect of the $\underline{3}$. drugs.

More important than an effect of steroids on the platelet count, of course, is an effect in preventing $\delta$ dangerous haemorrhage. Here the influence of $₹$ steroids in IM is unproved. There is anecdotal $\frac{0}{5}$ evidence that their use may reduce the time during which less serious haemorrhagic symptoms persist (Radel and Schorr, 1963) but fatal cerebral haemorrhage has occurred in IM despite steroid therapy o (Goldstein and Porter, 1969).

The authors do not suggest that steroids are ్ㅗㅇ inappropriate where major haemorrhage seems 0 likely in thrombocytopenic IM patients as there is clearly some evidence that they may, on occasion, $\frac{\mathrm{C}}{\mathbb{D}}$ influence the platelet count favourably; also the 울 effect of steroids in limiting bleeding is not mediated 0 only by elevation of the platelet count. These two

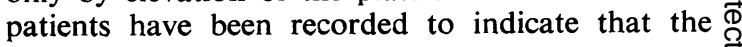


natural history of thrombocytopenia with bleeding in IM varies and includes episodes which, although severe, resolve with great rapidity. Efficacy of any drug in the management of thrombocytopenia in IM must be judged in the light of these observations.

\section{References}

ANGle, R.M. \& Alt, H.L. (1950) Thrombocytopenic purpura complicating infectious mononucleosis. Blood, 5, 449.

BenAIM, S. (1952) Infectious mononucleosis with thrombocytopenic purpura. British Medical Journal, 2, 1151.

Clarke, B.F. \& Davies, S.H. (1964) Severe thrombocytopenia in infectious mononucleosis. American Journal of the Medical Sciences, 248, 703.

Finlayson, R. (1951). Thrombocytopenic purpura complicating infectious mononucleosis. British Medical Journal, 2, 1563.

Freeman, T. \& Wakefield, G.S. (1958) Platelet agglutinating factor in glandular fever complicated by jaundice and thrombocytopenia. Lancet, ii, 883.

Goldbloom, A. \& Lieberson, A. (1948) A case of infectious mononucleosis with jaundice and thrombocytopenic purpura. American Journal of Medicine, 5, 912.

Goldstein, E. \& PoRTer, D.Y. (1969) Fatal thrombo- cytopenia with cerebral haemorrhage in infectious mononucleosis. Archives of Neurology, 20, 533.

Grossman, L.A. \& WolfF, S.M. (1959). Acute thrombocytopenic purpura in infectious mononucleosis. Journal of the American Medical Association, 171, 2208.

Krishnamurthy, M., L.ee, C.K. \& Dosik, H. (1976) Infectious mononucleosis and severe thrombocytopenia. American Journal of the Medical Sciences, 272, 221.

MyATT, A.V. \& LinN, R.H. (1957) Thrombocytopenia due to infectious mononucleosis-response to cortisone. Archives of Internal Medicine, 99, 139.

Ogilvie, C.M. \& Parry, T.E. (1952) Infectious mononucleosis with thrombocytopenia purpura. British Medical Journal, 2, 977.

RADEL, E.G. \& SCHORR, J.B. (1963) Thrombocytopenic purpura with infectious mononucleosis. Journal of Pediatrics, 63, 46.

ShaRP, A.A. (1969) Platelet bleeding and haemostasis in infectious mononucleosis. In: Infectious Mononucleosis (Ed by Carter, R. L. \& Penman, H.G.) p. 107. Blackwell Scientific Publications, Oxford.

VolPe, R., SPARKs, B.B. \& MAUTNER, L.S. (1953) Thrombocytopenic purpura complicating infectious mononucleosis. Canadian Medical Association Journal, 68, 269.

Weatherall, D.J. \& Bradley, J. (1974) The blood in systemic disease. In: Blood and its Disorders (Ed by Hardisty, R. M. \& Weatherall, D. J.), p. 1404. Blackwell Scientific Publications, Oxford. 